\title{
NOUVELLE
}

\section{Comment CD43 est-il exclu de la synapse immunologique?}

Jérôme Delon

tionnelles de ces modifications ont été analysées. Les résultats de ces expériences effectuées dans des modèles physiologiques [ 1 ,

Département de Biologie Cellulaire, Institut Cochin, 22, rue Méchain, 75014 Paris, France.
3] ont clairement établi que, dans ces conditions, CD43 n'était plus exclu de la synapse, démontrant que l'association CD43-ERM est
$>$ Trois articles parus dans le numéro du mois de novembre 2001 d'Immunity décrivent l'influence des protéines ERM (ezrineradixine-moésine) dans l'organisation de la synapse immunologique et dans la reconnaissance de l'antigène par les lymphocytes T [1-3].

La synapse immunologique se définit comme la région de contact étroit entre un lymphocyte $T$ et une cellule présentatrice de l'antigène (CPA) [4]. C'est grâce à ce type de contact intercellulaire que les peptides antigéniques présentés à la surface de la CPA sont reconnus par les récepteurs de l'antigène des lymphocytes $T$ (TCR). Cette zone se caractérise également par une accumulation de protéines impliquées dans la reconnaissance de l'antigène. Cependant, les mécanismes qui gouvernent la localisation précise de ces molécules restent largement inconnus. Les travaux rapportés ici proposent un modèle pour expliquer l'exclusion du CD43 de la synapse immunologique.

Les protéines ERM $[5,6]$ sont des molécules du cytosquelette qui agissent comme «molécule-pont» en liant le domaine cytoplasmique de certaines protéines transmembranaires telles que CD44, CD43, ICAM (intercellular adhesion molecule), Fas et L-sélectine aux microfilaments d'actine corticale localisés sous la membrane plasmique. Cependant, cette action n'est possible que si les protéines ERM sont phosphorylées au niveau d'un résidu thréonine particulier, localisé près de l'extrémité C-terminale (Thr 558 pour la moésine). La résolution partielle de la structure de la moésine par cristallogra- phie [7] suggère que les protéines ERM ainsi activées par phosphorylation adoptent une conformation ouverte qui leur permet de lier leurs partenaires présents sous la membrane plasmique. À l'inverse, en l'absence de phosphorylation, il est généralement admis que ces molécules sont inactivées par un repliement conformationnel qui induit une inhibition intramoléculaire des sites d'interaction des protéines ERM avec leurs ligands. Dans ce dernier cas, elles adoptent une localisation cytoplasmique.

L'analyse du rôle des protéines ERM dans la reconnaissance de l'antigène par les lymphocytes T, abordée dans les trois articles, repose principalement sur l'étude de la localisation subcellulaire de ces molécules par microscopie confocale. Plus précisément, il est montré que les protéines ERM sont exclues de la synapse immunologique. La liaison des protéines ERM à la molécule transmembranaire CD43 ayant été préalablement démontrée dans les lymphocytes T activés par des chimiokines [8], il n'a pas été surprenant de trouver que les protéines CD43 et ERM sont en fait coexclues en réponse à l'antigène. Cette indication ouvrait donc la voie à une hypothèse de travail particulièrement attrayante selon laquelle les protéines ERM pourraient servir à ancrer CD43 dans une zone éloignée de la synapse. Pour tester ce point, l'association ERM-CD43 a été perturbée par l'expression dans les lymphocytes $T$ d'un mutant dominant négatif de l'ezrine, ou d'un mutant de CD43 qui ne peut plus se lier aux protéines ERM, et les conséquences fonc- cruciale pour l'exclusion du CD43.

II restait donc à tester si cette localisation aberrante du CD43 avait une quelconque conséquence fonctionnelle sur la reconnaissance de l'antigène, ce qui permettrait de déterminer si l'exclusion du CD43 via son interaction avec les protéines ERM est vraiment cruciale pour l'activation $T$. Les résultats obtenus dans les différents articles ont été remarquablement homogènes : les événements précoces de l'activation $T$, que ce soit la formation de conjugués, les phosphorylations initiales sur tyrosines, la réponse calcique et le recrutement de la taline et de PKC-q sont normaux. En revanche, lorsque des événements plus tardifs ont été analysés, par exemple la production de cytokines, une nette inhibition de la synthèse d'interleukine (IL)-2 et d'interféron (IFN) $-\gamma$ a été démontrée dans les conditions où CD43 n'est pas exclu de la synapse. La molécule CD43 aurait donc un rôle inhibiteur, qui s'explique peutêtre par le fait que cette protéine possède un très grand domaine extracellulaire, fortement chargé négativement, et qui agit comme une barrière non spécifique gênant les interactions intercellulaires $[9,10]$. À l'inverse, ces expériences ont aussi révélé, ce qui était inattendu, que la présence continue de CD43 au niveau de la synapse n'entravait en rien la production d'autres cytokines comme I'IL-5 ou le TNF- $\alpha$. Ces différences de comportement peuvent s'expliquer si l'on prend en compte le fait que la présence du CD43 affecte surtout la phase soutenue de la signalisation déclenchée en réponse à l'activation du 
récepteur $T$ de l'antigène. Or, tous les facteurs de transcription ne répondent pas de la même façon à la durée de la signalisation $T$, et seuls ceux qui requièrent une activation soutenue seront touchés par l'effet inhibiteur du CD43, les conséquences de cette sensibilité se répercutant au niveau de leurs gènes cibles codant pour des cytokines.

Cependant, outre les changements de localisation subcellulaire des protéines ERM et leurs effets sur la position du CD43 dans les cellules $T$ interagissant avec une CPA, l'état d'activation de la moésine a aussi été analysé en mesurant le contenu en phospho-moésine dans les cellules $T$ par les techniques biochimiques classiques [1]. La moésine des lymphocytes T au repos a un niveau de phosphorylation de base sur Thr558 particulièrement élevé. Après reconnaissance de l'antigène par le lymphocyte T, une déphosphorylation rapide et massive de la moésine intervient dès la première minute de stimulation, puis le niveau de phosphorylation augmente à nouveau sans toutefois revenir à son niveau de base. Ces changements de phosphorylation ont été également visua-
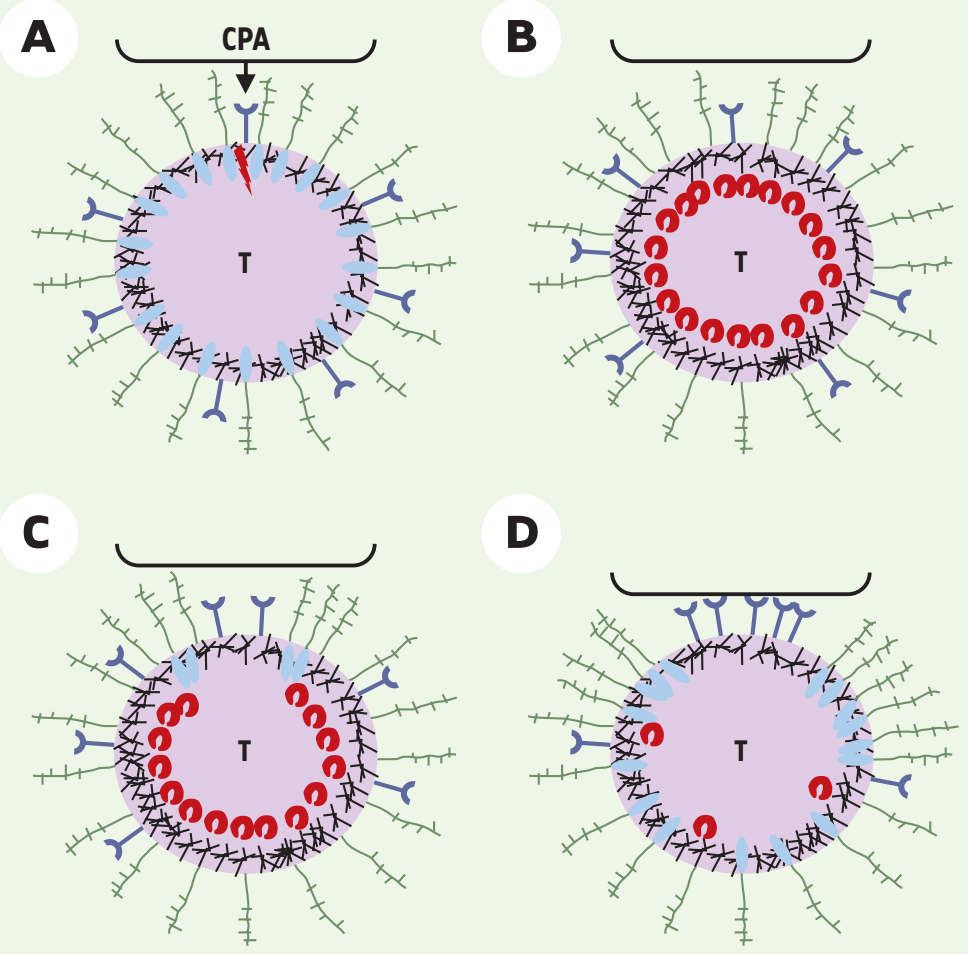

D
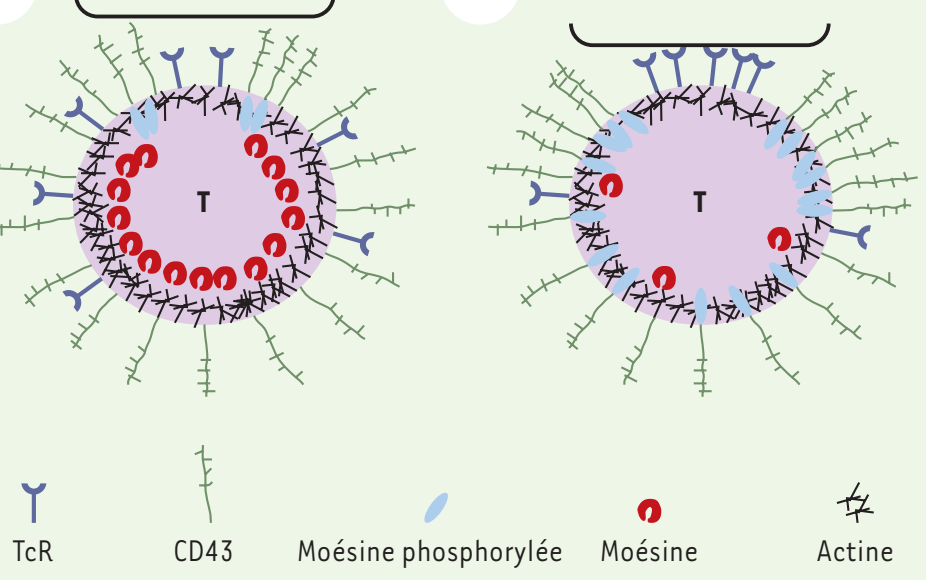

Figure 1. Modèle d'exclusion du CD43 de la synapse immunologique. Avant contact avec la CPA (A), les molécules de CD43 sont ancrées au cortex d'actine par leurs liaisons aux molécules de moésine phosphorylées. L'engagement d'une fraction des TcR est suffisant pour déclencher une déphosphorylation massive de la moésine (B). Les molécules de CD43, passivement exclues par l'interaction étroite des membranes des deux cellules, sont ré-ancrées à la périphérie de la synapse par une fraction de molécules de moésine qui est re-phosphorylée à cet endroit (C). Après une dizaine de minutes de contact, l'exclusion du CD43 est stabilisée par le ré-ancrage aux molécules de phospho-moésine (D).

lisés par microscopie sur cellule unique et corrélés à la capacité qu'ont les protéines ERM de se lier à CD43. Un modèle a ensuite été proposé pour intégrer l'ensemble de ces informations dans un schéma global qui explique comment les protéines ERM contrôlent l'exclusion du CD43 au niveau de la synapse immunologique (Figure 1). II est postulé que la déphosphorylation précoce des molécules de moésine, en libérant les grandes molécules de CD43 de tout ancrage au cortex d'actine, pourrait leur permettre de diffuser rapidement dans la membrane plasmique et d'être ainsi passivement exclues de la zone de contact entre la cellule T et la CPA. Néanmoins, ce mécanisme initial d'exclusion apparaît insuffisant pour maintenir CD43 à l'extérieur de la synapse. Le début de la vague de re-phosphorylation des molécules de moésine, touchant préférentiellement les molécules présentes à la périphérie du contact, pourrait assurer leur ré-ancrage stable dans une zone à présent exclue du centre de la synapse.

L'ensemble de ces travaux apporte donc de nouvelles informations sur l'organisation de la synapse immunologique. Les enzymes responsables des changements de phosphorylation de la moésine restent à préciser dans le contexte de la reconnaissance de l'antigène par les lymphocytes T. $\diamond$

Exclusion of CD43 from

the immunologic synapse

\section{REMERCIEMENTS}

À Grégoire Bonnet pour sa relecture du texte.

\section{RÉFÉRENCES}

1. Delon J, Kaibuchi K, Germain RN. Exclusion of CD43 from the immunological synapse is mediated by phosphorylation-regulated relocation of the cytoskeletal adaptor moesin. Immunity 2001 ; $15: 691-701$. 


\begin{tabular}{|c|c|c|c|c|}
\hline$\rightarrow \rightarrow \rightarrow$ & $\begin{array}{l}\text { 2. Roumier A, Olivo-Marin JC, } \\
\text { Arpin M, et al. The } \\
\text { membrane-microfilament } \\
\text { linker ezrin is involved in } \\
\text { the formation of the } \\
\text { immunological synapse } \\
\text { and in T cell activation. } \\
\text { Immunity } 2001 ; 15 \text { : } \\
715-28 \text {. } \\
\text { 3. Allenspach } \varepsilon \text { J, Cullinan P, } \\
\text { Tong J, et al. ERM- } \\
\text { dependent movement of } \\
\text { CD43 defines a novel } \\
\text { protein complex distal to } \\
\text { the immunological } \\
\text { synapse. Immunity } 2001 \text {; } \\
\text { 15: } 739-50 \text {. } \\
\text { 4. Delon J, Germain RN. }\end{array}$ & $\begin{array}{l}\text { Information transfer } \\
\text { at the immunological } \\
\text { synapse. Curr Biol } 2000 \text {; } \\
10 \text { : R923-33. } \\
\text { 5. Bretscher A, Chambers D, } \\
\text { Nguyen R, et al. ERM- } \\
\text { merlin and EBP50 protein } \\
\text { families in plasma } \\
\text { membrane organization } \\
\text { and function. Annu Rev Cell } \\
\text { Dev Biol } 2000 ; 16: 113-43 \text {. } \\
\text { 6. Gautreau A, Louvard D, } \\
\text { Arpin M. ERM proteins and } \\
\text { NF2 tumor suppressor: the } \\
\text { Yin and Yang of cortical } \\
\text { actin organization and cell } \\
\text { growth signaling. Curr Opin } \\
\text { Cell Biol } 2002 \text {; } 14: 104-9 \text {. }\end{array}$ & $\begin{array}{l}\text { 7. Pearson MA, Recezk D, } \\
\text { Bretscher A, et al. } \\
\text { Structure of the ERM } \\
\text { protein moesin reveals the } \\
\text { FERM domain fold masked } \\
\text { by an extended actin } \\
\text { binding tail domain. } \\
\text { Cell } 2000 ; 101: 259-70 \text {. } \\
\text { 8. Serrador JM, Nieto M, } \\
\text { Alonso-Lebrero JL, et al. } \\
\text { CD43 interacts with moesin } \\
\text { and ezrin and regulates its } \\
\text { redistribution to the } \\
\text { uropods of T lymphocytes } \\
\text { at the cell-cell contacts. } \\
\text { Blood } 1998 \text {; } 91: 4632-44 \text {. } \\
\text { 9. Cyster JG, Shotton DM, } \\
\text { Williams AF. The dimensions }\end{array}$ & $\begin{array}{l}\text { of the T lymphocyte } \\
\text { glycoprotein leukosialin } \\
\text { and identification of linear } \\
\text { protein epitopes that can } \\
\text { be modified by } \\
\text { glycosylation. EMBO J } \\
1991 ; 10: 893-902 \text {. } \\
\text { 10. Manjunath N, Correa M, } \\
\text { Ardman M, et al. } \\
\text { Negative regulation of } \\
\text { T-cell adhesion and } \\
\text { activation by CD43. Nature } \\
1995 \text {; } 377: 535-8 \text {. }\end{array}$ \\
\hline
\end{tabular}

\title{
NOUVELLE
}

\section{Anticorps anti-érythropoiétine chez des patients insuffisants rénaux traités par l'hormone recombinante}

Nicole Casadevall, Patrick Mayeux

\author{
N. Casadevall : Service d'Hématologie \\ Biologique, Hôtel-Dieu, \\ 1, place du Parvis de Notre Dame, \\ 75181 Paris Cedex 04, France. \\ P. Mayeux : Département d'Hématologie, \\ Institut Cochin, 27, rue du Faubourg \\ Saint-Jacques, 75014 Paris, France.
}

L'érythropoïétine ( $\varepsilon$ po) est indispensable à la formation des globules rouges et l'invalidation des gènes codant pour l'Epo ou son récepteur ( $\varepsilon p \circ R)$ provoque une anémie létale chez la souris au stade fœtal [1]. L'Epo est principalement produite par le rein chez l'animal adulte et, dans une faible proportion, par le foie. L'anémie observée chez les insuffisants rénaux est en grande partie due à une forte diminution de la production rénale d'Epo. Toutefois, la production hépatique d'hormone, et probablement une production rénale résiduelle, permettent de maintenir une certaine érythropoïèse médullaire. La mise sur le marché d'érythropoïétine recombinante à la fin des années 1980 a permis de traiter l'anémie de ces patients dans les pays occidentaux et a supprimé leurs besoins transfusionnels et, du même coup, tous les risques et inconvénients associés à cet acte. L'Epo recombinante est également prescrite lors d'anémies associées à d'autres pathologies comme les cancers, ou celles qui sont observées lors d'actes chirurgicaux. Elle est également utilisée dans des protocoles d'autotransfusions programmées.

La forme circulante de l'Epo est une molécule de 165 acides aminés avec une structure tridimensionnelle très stable formant quatre hélices $\alpha$. La molécule est fortement glycosylée, sa masse moléculaire totale est d'environ $34 \mathrm{kDa}$, la masse moléculaire de la partie protéique est de 18,3 kDa. L'Epo est fortement conservée chez les mammifères, comme en témoignent les $80 \%$ d'identité au niveau protéique de l'Epo humaine et murine, et une activité biologique totalement conservée d'une espèce à l'autre. Deux types d'Epo humaine recombinante sont commercialisées actuellement en France - l'Epo $\alpha$ et l'Epo $\beta$ - qui ne diffèrent qu'au niveau de la glycosylation : l'Epo $\alpha$ portant davantage d'acides sialiques que l'Epo $\beta$.
Malgré la grande conservation des séquences, l'Epo est une molécule très antigénique et l'injection d'Epo recombinante humaine à un animal provoque quasi systématiquement l'apparition d'anticorps neutralisants. Dans la majorité des cas, ces anticorps neutralisent aussi l'Epo endogène de l'animal et conduisent à des érythroblastopénies sévères. Néanmoins, le traitement par l'Epo recombinante est remarquablement bien toléré et, depuis sa mise sur le marché jusqu'en 1997, seuls trois cas d'apparition d'anticorps neutralisants ont été rapportés dans le monde [2-4]. Cependant, depuis 4 ans nous avons détecté l'apparition d'anticorps neutralisants chez 34 patients traités par l'Epo recombinante. Un article récemment publié décrit les treize premiers cas identifiés [5]. Tous ces patients avaient été traités par l'Epo recombinante pour des anémies 